\title{
Philae's comet discoveries create series of conundrums
}

\section{Lander may never be heard from again, but latest data haul throws up plenty of mysteries.}

\section{Elizabeth Gibney}

30 July 2015 | Updated: 03 August 2015
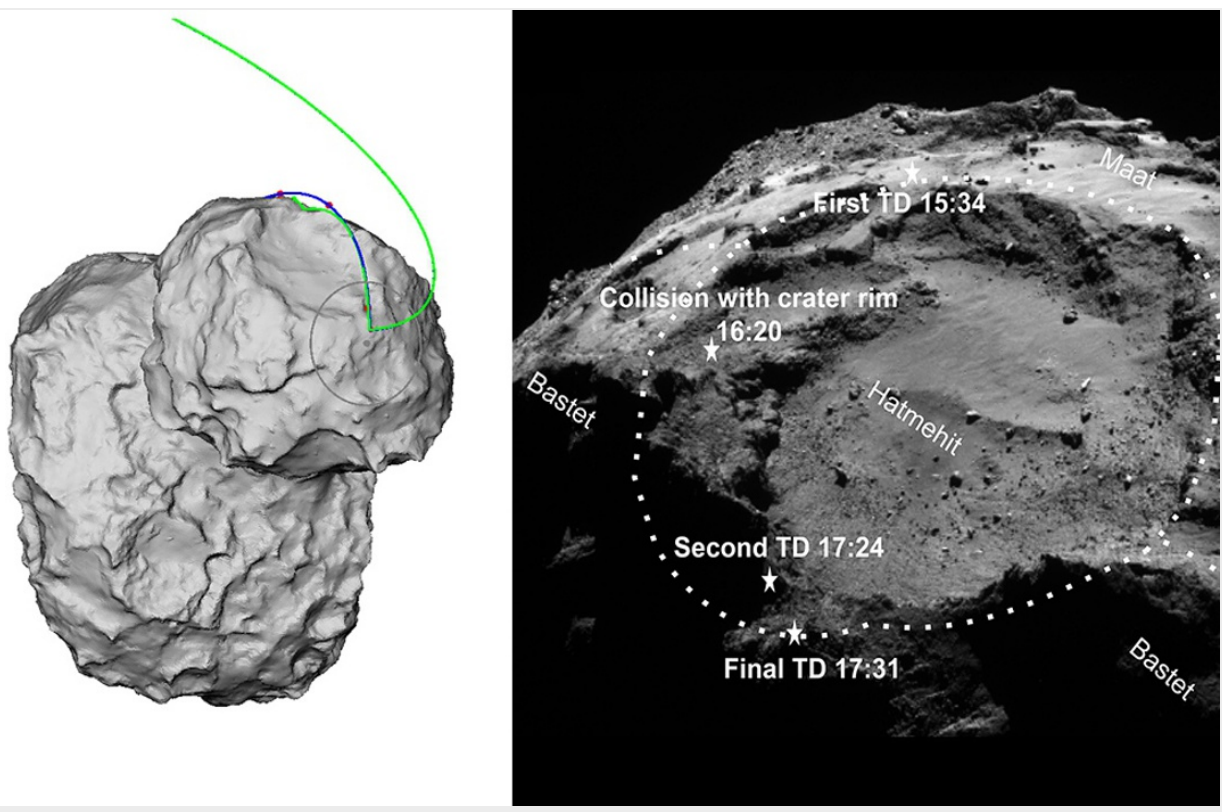

ESAROSETTASSONC

The Philae lander followed a curved trajectory (green) before landing on comet 67P/Churyumov-

Gerasimenko and bouncing twice (right).

The Philae comet lander has not spoken to Earth since a link-up through its parent spacecraft, Rosetta, on 9 July — and scientists may never hear from it again.

But a haul of seven reports analysing data that Philae collected and sent home over three days in November last year, before it went into hibernation, has revealed further puzzling information about the comet it sits on, which is whirling closer to the Sun. Some scientists say the findings suggest that the comet is not an unaltered time capsule from the dawn of the Solar System, as researchers had presumed.

"It seems like the more we know, the less we know," says Geraint Morgan, a co-investigator on the lander's Ptolemy instrument and a physical chemist at the Open University in Milton Keynes, UK. "The comet is more complicated than we might have imagined."

\section{The hard problem}

One puzzle is that the surface of comet 67P/Churyumov-Gerasimenko is much harder than scientists had expected, according to papers published in Science on 30 July. When Philae landed on 12 November 2014, it bounced, grazed the lip of a crater, and bounced again before coming to rest. Measurements of how its legs compressed as they hit the comet ${ }^{1}$, as well as data from a hammer on Philae ${ }^{2}$ that tried unsuccessfully to penetrate the surface, show that the comet has a strong, hard crust, covered in places by a softer layer of dust and ice.

That finding could change future lander design, says Stephan Ulamec, project manager for the lander at the German Aerospace Center (DLR) in Cologne. Before the mission, some had feared that the lander might sink into several metres of soft dust. "In the future one would have to think about a mechanism that can cope with pretty hard material," he says.

The hard surface could be the result of ice grains either compacting or crystallizing under solar radiation. Comet simulations in the 1990s showed that these processes were possible, says Karsten Seiferlin, a planetary scientist at the University of Bern and a member of Philae's hammer team. 
That the comet could have changed since its formation has implications for the wider mission, which had assumed that such bodies had existed largely unaltered since the start of the Solar System, says Seiferlin. But both the hard crust and a large variety of surface materials and structures found on the comet could be the result of recent modifications, he says.

For instance, an intriguing polymer found in the dust ${ }^{3}$ — called polyoxymethylene - may form when sunlight hits the comet's surface and triggers reactions that link simple formaldehyde molecules into chains, researchers suggest. If the same kind of process is producing other complex organic molecule at the comet's surface, this could explain its dark colour (though polyoxymethylene itself is white), says Andrew Morse, a member of the Ptolemy team and a planetary scientist at the Open University. The polymer may also be masking signals from other interesting compounds formed earlier in the comet's history, he adds.

"It means we have to understand the physics and processes going on if we want to draw conclusions about the history of the Solar System," says Seiferlin.

\section{Rugged landscape}

Images gathered by Philae show that Comet 67P has a varied and fractured surface, with changes in texture and reflectivity ${ }^{4}$, as well as boulders and features shaped by erosion ${ }^{5}$. Since features like this were first observed, researchers have wondered what caused such a disparate landscape. Data from the lander's CONSERT experiment, which probes the comet's interior by sending radio waves between Philae and the Rosetta orbiter, have deepened the puzzle: they show a fairly uniform composition inside the 'head' of the rubber-duck-shaped comet, at least on a scale of tens of metres ${ }^{6}$.

The data also show the comet to be very porous - between $75 \%$ and $85 \%$ empty space. The comet's low-density is not incompatible with its hard surface, but how to reconcile them will take some explaining, says Michael A'Hearn, an astronomer at the University of Maryland in College Park who is part of the team running the orbiter's OSIRIS camera.

Philae has found organic compounds on the surface of 67P, including alcohols and

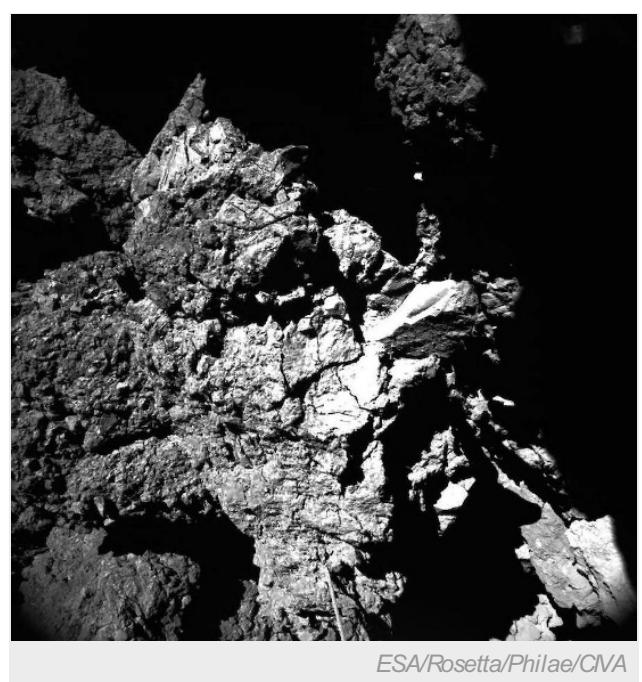

The ground at Philae's landing site, Abydos. amines ${ }^{7}$ and four substances never before found on a comet. Although the results are exciting for being the first from the surface of a comet, they are not particularly surprising, says Fred Goesmann of the Max Planck Institute for Solar System Research in Munich, Germany, who is principal investigator for a chemical-analysing instrument, COSAC. Such compounds could be the starting materials from which life grows, but the lander found no evidence that comets such as 67P brought the ingredients of life to Earth, as some scientists hypothesize. Neither COSAC nor Ptolemy, which is designed to detect light elements, obtained drilled samples. Instead both tested their samples only in a simplified 'sniffing' mode, which meant that COSAC could not analyse the 'handedness', or chirality, of molecules to see whether they matched that of life on Earth; nor could Ptolemy compare the ratios of different isotopes in comet samples with those on Earth.

\section{Last rites?}

The teams were lucky to get anything: the data come from a sample of dust accidentally kicked up into the lander's exhaust on its initial touchdown, and analysed in mid-air. Sniffing data from Philae's less dusty final resting site are more scarce. If Philae were to take data now, even in sniffing mode, the comet's increased release of gas and dust as it flies closer to the Sun could mean a much greater haul, but scientists are not confident that they will hear anything more. Although warmer and sunnier conditions allowed Philae to reboot its solar-powered batteries in June, the lander has spoken to Earth on only a handful of occasions. Rosetta's own science mission has now taken it to the comet's southern hemisphere and out of site for Philae, and the next opportunity to communicate will not be until 7 August, says Cinzia Fantinati, Philae operations manager at the DLR.

Researchers believe that at least one of the lander's transmitters has degraded, and data on the illumination of its solar panels suggest that either the craft has moved slightly, or the terrain around it has shifted. Rosetta and Philae's antennas must be carefully aligned if they are to communicate, but combinations that were successful in June no longer seem to work. The researchers have attempted to blind-activate a series of commands sent in November, which would tell Philae to carry out some simple science tasks; it could then store the data in the hope that a stable connection can be established, says Fantinati. Patrick Martin, Rosetta's mission manager, says that the European Space Agency mission will now focus its scientific priority on the orbiter — which is currently studying the comet's southern hemisphere - although it will keep attempting to contact the lander. 
Nature | doi:10.1038/nature.2015.18102

\section{Updates}

Updated:This article has been updated. Andrew Morse originally told Nature's news team that poloxymethylene might explain the comet's dark colour. Upon reflection, he notes that the polymer itself could not be responsible for the colour - but its presence suggests that other organic molecules are likely forming through similar processes, and that these might give rise to the comet's dark surface.

\section{References}

1. Biele, J. et al. Science http://dx.doi.org/10.1126/science.aaa9816 (2015).

2. Spohn, T. et al. Science http://dx.doi.org/10.1126/science.aab0464 (2015).

3. Wright, I. P. et al. Science http://dx.doi.org/10.1126/science.aab0673 (2015).

4. Bibring, J.-P. et al. Science http://dx.doi.org/10.1126/science.aaa0671 (2015).

5. Mottola, S. et al. Science http://dx.doi.org/10.1126/science.aab0232 (2015).

6. Kofman, W. et al. Science http://dx.doi.org/10.1126/science.aab0639 (2015).

7. Goesmann, F. et al. Science http://dx.doi.org/10.1126/science.aab0689 (2015). 